\title{
Efeito da idade das larvas de Ceratitis capitata (Wied.) sobre a qualidade biológica do parasitoide Diachasmimorpha longicaudata (Ashmead)
}

\author{
Effect of the age of larvae Ceratitis capitata (Wied.) in the biological \\ quality of parasitoid Diachasmimorpha longicaudata (Ashmead)
}

\author{
Patrícia Cristina do Carmo Oliveira', Edna Dias Fonseca', Clarice Diniz Alvarenga * \\ Terezinha Augusta Giustolin', Marcelo Mendes Rabelo', Cristiane Ramos Coutinho'
}

| | | | | | | | | | | | | | | | | | | | | | | | | | | | | | | | | | | | | | | | | | | | | | | | | | | | | | | | | | | | | | | | | | | | | | | | | | | | | | | | | | | | | | | | | | | | | | | | | | | | | | | | | | | | | | | | | | | | | | | | | | | | | | | | | | | | | | | | | | | | | | | | | | | | | | | | | | | | | | | | | | | | | | | | | | | | | | | | | | | | | | | | | | | | | | | | | | | |

\begin{abstract}
RESUMO: Objetivou-se avaliar a influência da idade (tamanho) das larvas de Ceratitis capitata utilizadas na criaçáo de Diachasmimorpha longicaudata sobre o parasitismo, a razão sexual e a longevidade das fêmeas da progênie, quando as fêmeas mães foram mantidas sozinhas ou competindo pelo mesmo hospedeiro. Para isso, foram oferecidas larvas de cinco, seis, sete e oito dias de idade às fêmeas do parasitoide em "unidades de parasitismo", no interior de gaiolas adaptadas, contendo uma ou duas fêmeas do parasitoide de sete dias de idade. O número de fêmeas do parasitoide dividindo a mesma "unidade de parasitismo" não afetou nenhum aspecto da qualidade do parasitoide. O tamanho do hospedeiro influenciou significativamente no número de parasitoides emergidos. Larvas hospedeiras menores (cinco dias) provocaram uma redução no parasitismo em relação às larvas de seis, sete e oito dias de idade. Houve maior emergência de parasitoides machos de hospedeiros com cinco e seis dias de idade, enquanto mais fêmeas emergiram de larvas com sete e oito dias de idade. As fêmeas de D. longicaudata provenientes de larvas com seis, sete e oito dias viveram mais do que as fêmeas obtidas de larvas com cinco dias de idade. Larvas hospedeiras maiores, quando utilizadas na criação de parasitoides de moscas-das-frutas, aumentam a porcentagem de parasitismo, o número de fêmeas na progênie e a longevidade das fêmeas da progênie, podendo proporcionar vantagens econômicas quando utilizadas para liberaçáo aumentativa no campo.
\end{abstract}

PALAVRAS-CHAVE: controle biológico; parasitismo; razão sexual; Braconidae; Tephritidae.

\begin{abstract}
This work was carried out in order to evaluate the influence of age (size) of larvae Ceratitis capitata used in the rearing of the Diachasmimorpha longicaudata on parasitism, sex ratio of the progeny and the longevity of progeny's females, when the mothers were maintained alone or sharing the same host. Larvae at different ages (five, six, seven and eight days old), in "units of parasitism" were offered to female parasitoids, inside adapted cages, containing one or two seven days old female parasitoids. The number of females of the parasitoid sharing the same "unit of parasitism" in the same cage did not affect any aspect of parasitoid quality. However, the size of the host had significant influence on the number of emerged parasitoids. The youngest host larvae (five days old) reduced parasitism more than larvae at six, seven and eight days old. There was higher emergence of male parasitoids of five and six days old hosts, while more females emerged from seven and eight days old larvae. The females of $D$. longicaudata from larvae with six, seven and eight days old lived more than those obtained from females of five days old larvae. When larger host larvae are used in the rearing of parasitoids of fruit flies, it increases parasitism, the number of female progeny and the longevity of female progeny; it can provide economic advantages when used for augmentative liberation on the field.
\end{abstract}

KEYWORDS: biological control; parasitism; sex ratio; Braconidae; Tephritidae. 


\section{INTRODUÇÃO}

As moscas-das-frutas (Diptera: Tephritidae) são as principais pragas das frutíferas em todo o mundo, e vêm causando grandes prejuízos principalmente pelos danos diretos causados na polpa dos frutos. Segundo Godoy et al. (2011), anualmente são perdidos no mundo todo cerca de 1 bilhão de dólares devido aos danos causados por essas moscas. No Brasil se destacam as espécies do gênero Anastrepha (Schiner) e a espécie Ceratitis capitata (Wiedemann).

O controle dessas pragas vem sendo feito principalmente pelo manejo químico, mas, atualmente, os programas de manejo integrado têm se destacado, incentivando o desenvolvimento e a utilização de outros métodos. Dentre eles estão os métodos culturais, a utilização de substâncias atrativas, a técnica do inseto estéril (TIE), o uso da resistência varietal e, principalmente, o controle biológico. O parasitoide Diachasmimorpha longicaudata (Ashmead) (Hymenoptera: Braconidae) tem sido o agente de controle biológico aumentativo de moscas-das-frutas mais estudado e utilizado mundialmente. Seu destaque se deve à rápida adaptação aos meios onde ele é liberado, por ser um parasitoide específico de tefritídeos e pela facilidade com que é criado em laboratório (Garcia; Ricalde, 2012).

O sucesso dos programas de controle biológico depende de estudos básicos sobre os inimigos naturais, os quais devem ser criados e liberados em grandes quantidades no campo. Contudo, pouco se conhece sobre a relação do parasitoide com seu hospedeiro. Sabe-se que para garantir o sucesso na produção de $D$. longicaudata em criaçấo massiva, a qualidade das larvas hospedeiras é um dos requisitos indispensáveis para assegurar bons resultados de controle.

O tamanho do hospedeiro pode ter efeito importante sobre o comportamento e a biologia do parasitoide D. longicaudata (Eben et al., 2000; López et al., 2009; Montoya et al., 2011). Geralmente, a fêmea do parasitoide faz a seleçáo do tamanho do hospedeiro para realizar a postura de seus ovos, de forma a depositar seus ovos fecundados (produzem fêmeas e machos) em hospedeiros maiores, e ovos não fecundados (produzem machos somente) em hospedeiros menores (Godfray, 1994).

O tamanho das larvas hospedeiras pode ser um fator útil para determinar a qualidade dos parasitoides produzidos, já que possivelmente afeta aspectos da qualidade do inimigo natural, como a razáo sexual da progênie. Para insetos parasitoides, o número de fêmeas produzidas por hospedeiro é um indicador de sua capacidade como agente de controle biológico (Lopez et al., 2009). Quanto maior o número de fêmeas produzidas, mais eficiente e barata será a sua produção, e mais acessível será a sua utilização nos programas de controle biológico. Portanto, o objetivo deste trabalho foi avaliar o efeito da idade das larvas de C. capitata nos aspectos biológicos de D. longicaudata.

\section{MATERIAL E MÉTODOS}

Os ovos de C. capitata e o parasitoide D. longicaudata utilizados nos experimentos foram obtidos de criaçóes estoques, mantidas em condiçôes controladas de temperatura, umidade e luz $\left(26 \pm 1^{\circ} \mathrm{C}, 65 \pm 10 \%\right.$ de UR e fotofase de 14 horas).

Para a obtençáo das larvas de C. capitata de diferentes idades (larvas com cinco, seis, sete e oito dias de idade), diariamente, durante quatro dias, foram distribuídos ovos de C. capitata em recipientes plásticos contendo dieta artificial para larvas, segundo a metodologia descrita por CARVALHO et al. (1998). Os recipientes foram fechados com filme plástico e mantidos em câmara B.O.D., regulada a $26 \pm 1^{\circ} \mathrm{C}$, e fotoperíodo de 14 horas, onde permaneceram até a larva atingir a idade desejada.

Para a realização dos experimentos, adultos de D. longicaudata com quatro dias de vida foram retirados das gaiolas de criação estoque e transferidos para gaiolas adaptadas. Essas gaiolas foram confeccionadas a partir de potes plásticos transparentes com tampa e capacidade para $500 \mathrm{~mL}$. Nas tampas foi feito um corte circular, onde foi colocado um tecido fino do tipo voil para permitir a entrada de ar e evitar a fuga dos insetos. Em cada gaiola foi colocado um ou dois casais do parasitoide, que foram alimentados com dieta artificial à base de mel e água, seguindo a metodologia proposta por Carvalho et al. (1998).

Para evitar alguma influência da presença do macho no comportamento das fêmeas no momento do parasitismo, estes foram mantidos nas gaiolas somente por dois dias para ocorrer a cópula, e, posteriormente, foram retirados. Oito dias depois, a contar da primeira coleta de ovos de C. capitata, as larvas foram expostas ao parasitismo. Para tanto, foram construídas "unidades de parasitismo", que simulam frutos infestados, conforme metodologia de Carvalho et al. (1998). Estas unidades foram confeccionadas a partir de um tecido fino do tipo voil, utilizado para envolver um lote de larvas de C. capitata. Juntamente às larvas, na "unidade de parasitismo", colocou-se uma pequena quantidade de dieta para elas se alimentarem, uma vez que nem todas haviam atingido o $3^{\circ}$ instar. Em cada "unidade de parasitismo" foram colocadas 20 larvas (em gaiolas contendo uma fêmea) ou 40 larvas (em gaiolas com duas fêmeas).

As "unidades de parasitismo" contendo as larvas foram penduradas nas gaiolas e oferecidas ao parasitismo durante duas horas. Após este período, as larvas de C. capitata com oito dias de idade foram transferidas diretamente para recipientes contendo vermiculita umedecida e mantidas até a emergência dos adultos. As larvas de cinco, seis e sete dias de idade foram transferidas para recipientes contendo dieta artificial, que foram fechados com voil e presos com elástico. Após atingirem o terceiro instar, as larvas da dieta também foram transferidas para recipientes contendo vermiculita, visando à emergência dos adultos de D. longicaudata. Os machos e fêmeas dos adultos emergidos 
foram contabilizados, visando ao cálculo da razão sexual e a porcentagem de parasitismo. A razão sexual foi calculada conforme a fórmula: número de fêmeas/(número de machos + número de fêmeas).O índice de parasitismo (IP) foi calculado conforme Carvalho (2003).

Após a emergência de $D$. longicaudata, os machos foram descartados e as fêmeas foram transferidas para gaiolas sem água e alimento (simulando uma situação provável em campo). As gaiolas contendo cinco fêmeas cada foram mantidas no laboratório, dispostas em estantes de aço, sob condiçôes controladas $\left(26 \pm 1^{\circ} \mathrm{C}, 65 \pm 10 \%\right.$ de UR e fotofase de 14 horas). Foi avaliada a mortalidade diária, e as fêmeas mortas foram retiradas.

\section{Análise estatística}

O delineamento utilizado foi o inteiramente casualizado, em esquema fatorial $4 \times 2$ para as variáveis, índice de parasitismo e razão sexual das fêmeas de D. longicaudata, considerando quatro idades das larvas de C. capitata (larvas de cinco, seis, sete e oito dias de idade), duas quantidades de fêmeas na gaiola (uma ou duas fêmeas/gaiola) e dez repetiçôes. Para a variável longevidade das fêmeas da progênie foi utilizado o esquema fatorial 4 × 2, sendo quatro idades das larvas de C. capitata (larvas de cinco, seis, sete e oito dias de idade), duas quantidades de fêmeas e cinco repetiçôes. Os dados foram analisados (ANOVA) e as médias foram comparadas pelo teste Scott-Knott a $5 \%$ de probabilidade, utilizando o programa Sisvar (Ferreira, 2000), e, quando necessário, os dados foram transformados em $\sqrt{ } \mathrm{x}+0,5$. Todas as tabelas apresentaram as médias originais.

\section{RESULTADOS E DISCUSSÃO}

Não houve interação entre a idade das larvas e o número de fêmeas de $D$. longicaudata para nenhuma das causas da variação (índice de parasitismo, longevidade das fêmeas da progênie do parasitoide e razão sexual da progênie do parasitoide).

A presença de mais de uma fêmea do parasitoide na gaiola não influenciou a razão sexual da progênie de $D$. longicaudata. A razão sexual da progênie foi influenciada apenas pela idade das larvas de C. capitata (Tabela 1). Um maior número de machos emergiu de larvas com cinco e seis dias de idade, indicado pelos valores de razáo sexual obtidos abaixo de 0,5 . Já quando as larvas de $C$. capitata estavam com idades de sete e oito dias, a razáo sexual obtida foi superior a 0,5 , ou seja, houve emergência de maior número de fêmeas.

Esse resultado indica que utilizar um maior número de larvas de C. capitata com menor tamanho levará à maior emergência de machos na criação, não sendo o mais indicado para um programa de produçáo massal deste parasitoide. A influência da idade do hospedeiro já foi avaliada por outros autores, visando à melhoria na qualidade final do parasitoide a ser liberado no campo para o controle biológico de espécies de moscas-das-frutas. VAn Nieuwenhove; Ovruski (2011), buscando determinar a idade ideal para expôr as larvas de Anastrepha fraterculus (Wied.) à D. longicaudata, observaram que a melhor fase larval foi da metade do ciclo até o final do terceiro instar, quando as larvas apresentavam idades de 9 a 12 dias. Os autores relataram que emergiram mais fêmeas das larvas de terceiro instar e mais machos de larvas de segundo instar. Estes resultados foram semelhantes aos obtidos neste trabalho, quando foram avaliadas como hospedeiro as larvas de C. capitata.

Além disso, em alguns casos, diferentes espécies hospedeiras podem influenciar a razão sexual da progênie por possuírem tamanhos diferentes, como é o caso de Anastrepha ludens (Loew) e Anastrepha obliqua (Macquart), criadas em manga (Mangifera indica L.) (EBEN et al., 2000). Maior proporçāo de fêmeas foi observada quando $D$. longicaudata foi criado a partir de $A$. ludens, as quais possuem larvas maiores. A diferença foi ainda mais evidente quando se utilizou $C$. capitata e A. fraterculus. Ovruski et al. (2011) observaram que a proporçáo de descendentes do sexo feminino de $D$. longicaudata foi sempre significativamente maior quando o parasitoide foi criado em $A$. fraterculus do que quando foi criado em larvas de C. capitata.

O tamanho do hospedeiro influencia a fêmea de $D$. longicaudata no momento do parasitismo, a ponto de esta selecionar o hospedeiro de forma a contornar a falta de recursos para a progênie, depositando seus ovos fecundados em hospedeiros maiores para, assim, produzir fêmeas mais vigorosas nesses hospedeiros (Godfray, 1994). Essa escolha deve ser comandada por algum estímulo proveniente das larvas hospedeiras, quando se encontram maiores e nutricionalmente adequadas. DuAn; Messing (2000) comentaram, ao avaliarem $D$. longicaudata, que os sinais químicos produzidos pelas

Tabela 1. Médias ( \pm Erro Padrão) da razão sexual da progênie de Diachasmimorpha longicaudata sobre larvas de Ceratitis capitata de diferentes idades. Temperatura $26 \pm 1{ }^{\circ} \mathrm{C}$; UR $65 \pm$ $10 \%$ e Fotofase $14 \mathrm{~h}$.

\begin{tabular}{lccc}
$\begin{array}{lccc}\text { Idade } \\
\text { da larva } \\
\text { (dias) }\end{array}$ & $\begin{array}{c}\text { Número de fêmeas por } \\
\text { "unidade de parasitismo" }\end{array}$ & Média \\
\cline { 1 - 3 } 8 & 1 & 2 & \\
\cline { 1 - 3 } & $0,63 \pm 0,09$ & $0,53 \pm 0,58$ & $0,58 \pm 0,05 \mathrm{a}$ \\
\hline 7 & $0,56 \pm 0,07$ & $0,51 \pm 0,09$ & $0,51 \pm 0,05 \mathrm{a}$ \\
\hline 6 & $0,29 \pm 0,09$ & $0,20 \pm 0,04$ & $0,24 \pm 0,06 \mathrm{~b}$ \\
\hline 5 & $0,08 \pm 0,03$ & $0,17 \pm 0,09$ & $0,13 \pm 0,05 \mathrm{~b}$ \\
\hline Média & $0,39 \pm 0,04 \mathrm{~A}$ & $0,33 \pm 0,04 \mathrm{~A}$ & \\
\hline CV & \multicolumn{3}{c}{14,13} \\
\hline
\end{tabular}

Médias seguidas de mesma letra, maiúscula na linha e minúscula na coluna, não diferem entre si pelo Teste de Scott Knott, com 5\% de probabilidade. 
larvas de C. capitata tiveram pouca influência sobre o comportamento do parasitoide em relação à razão sexual da progênie.

Alguns autores náo concordam que o tamanho do hospedeiro influencia diretamente na proporçáa do sexo de $D$. longicaudata, mas sim que a proporção de machos e fêmeas é influenciada pela intensidade de superparasitismo. Montoya et al. (2011, 2012), por exemplo, observaram que o superparasitismo foi positivamente correlacionado com o comprimento da larva hospedeira. Verificando uma relação entre o tamanho do hospedeiro e o superparasitismo no aumento da proporção de fêmeas, os autores observaram que o tamanho do hospedeiro não influenciou significativamente a proporçáo de machos e fêmeas, mas constataram que essa proporção foi afetada significativamente pela intensidade de parasitismo. Segundo os mesmos autores, as fêmeas realizam mais posturas em hospedeiros maiores, levando à tendência da emergência de fêmeas. Provavelmente isso possa ter ocorrido no presente trabalho, porém, como a intensidade de superparasitismo é avaliada pelo número de cicatrizes deixadas pelas fêmeas no momento da postura, não é possível afirmar esta hipótese, já que nấo foi registrado o número de cicatrizes nas larvas parasitadas neste estudo.

A idade do hospedeiro também afetou significativamente o número de parasitoides emergidos, e, novamente, o número de fêmeas na gaiola não afetou o índice de parasitismo de D. longicaudata (Tabela 2). Todas as idades das larvas de C. capitata promoveram índices de parasitismo que variaram de 55,0 a 58,8\%, com exceção das larvas com cinco dias de idade, nas quais o índice de parasitismo foi significativamente inferior $(37,8 \%)$.

O menor parasitismo ocorrido nas larvas de cinco dias de idade corrobora os resultados obtidos por OvRUsKi et al. (2011). Os autores avaliaram a preferência de D. longicaudata por larvas de C. capitata e A. fraterculus e constataram que, apesar de ter ocorrido parasitismo das larvas de C. capitata e de $A$. fraterculus, quando a fêmea teve a chance de escolha, preferiu parasitar $A$. fraterculus. Segundo os autores, a preferência está relacionada ao tamanho da larva hospedeira, uma vez que as larvas de $A$. fraterculus são duas vezes maiores que as larvas de C. capitata.

A reduçáo no parasitismo de $D$. longicaudata, quando foram utilizadas larvas de C. capitata com cinco dias de idade, pode ter sido causada pelo menor tamanho dessas larvas, pois, devido à menor idade, apresentavam condiçóes nutricionais inferiores às demais larvas avaliadas. LAWRENCE $(1982,1986)$ relatou que as larvas de primeiro instar de $D$. longicaudata necessitam de um ambiente com adequaçáo hormonal no interior do hospedeiro para avançar para o segundo ínstar, o que ocorre quando as larvas do hospedeiro pupam. No presente trabalho as larvas do parasitoide morreram, resultando em baixas emergências de $D$. longicaudata, já que não havia este ambiente favorável às larvas do parasitóide. VAN Nieuwenhove; OvRuski (2011) dissecaram as pupas de $A$. fraterculus não emergidas que foram expostas ao parasitismo quando as larvas estavam no segundo instar, e constataram que cerca de $86 \%$ continham cadáveres de ovos e larvas de primeiro instar de $D$. longicaudata.

Outra hipótese para o menor parasitismo nas larvas menores pode estar relacionado ao observado por LópEZ et al. (2009), que constataram que o parasitismo de $D$. longicaudata foi maior em larvas de $A$. fraterculus de tamanho médio em comparaçáo a larvas de tamanho grande, e citam três prováveis fatores que podem ter levado a tal resultado: larvas maiores eram menos abundantes no lote exposto ao parasitismo, e isso pode ter afetado a probabilidade de serem encontradas para a oviposiçáo pelas fêmeas; as larvas maiores podem ter mais mobilidade do que as pequenas e médias, o que lhes permitiu escapar do parasitismo; larvas maiores podem ser mais maduras e não serem hospedeiros apropriados para o desenvolvimento do inseto.

No entanto, Wong; Ramadan (1992) mostraram que o pico de emergência de $D$. longicaudata foi alcançado durante o segundo instar de Bactrocera dorsalis (Hendel), reduzindo quando os hospedeiros estavam maiores, perto da pupaçáo. Fato semelhante pode ter ocorrido neste estudo, quando as larvas poderiam não estar perto do estágio de pupa. Por isso, não houve redução na emergência do parasitoide, no entanto, também não diferiu das larvas menores (seis dias). Outra hipótese possível está relacionada à não preferência do parasitoide pela larva hospedeira. Isso faria com que as fêmeas realizassem a oviposição em qualquer estádio larval. Entretanto, no momento da oviposiçẫo haveria uma seleção pelas fêmeas do parasitoide para ovipositar seus ovos fecundados em larvas hospedeiras mais vigorosas, favorecendo a progênie fêmea da espécie. Assim, apesar de não ter ocorrido diferença quanto ao parasitismo entre as larvas de seis, sete e oito dias de idade (Tabela 2), as larvas de sete e oito dias proporcionaram a emergência de maior número de fêmeas, o que é de suma importância para o controle biológico, uma vez que o macho não controla as moscas-das-frutas.

Tabela 2. Médias ( \pm Erro Padrão) do Índice de Parasitismo (\%) de Diachasmimorpha longicaudata sobre larvas de Ceratitis capitata de diferentes idades. Temperatura $26 \pm 1{ }^{\circ} \mathrm{C}$; UR $65 \pm 10 \%$ e fotofase $14 \mathrm{~h}$.

\begin{tabular}{|c|c|c|c|}
\hline \multirow{2}{*}{$\begin{array}{l}\text { Idade } \\
\text { da larva } \\
\text { (dias) }\end{array}$} & \multicolumn{2}{|c|}{$\begin{array}{l}\text { Número de fêmeas por } \\
\text { "unidade parasitismo" }\end{array}$} & \multirow{2}{*}{ Média } \\
\hline & 1 & 2 & \\
\hline 8 & $54,02 \pm 5,96$ & $59,24 \pm 4,99$ & $56,63 \pm 6,64 a$ \\
\hline 7 & $59,10 \pm 7,54$ & $57,56 \pm 7,67$ & $58,34 \pm 8,45 a$ \\
\hline 6 & $52,72 \pm 9,21$ & $57,80 \pm 9,22$ & $55,27 \pm 8,01 a$ \\
\hline 5 & $38,13 \pm 6,50$ & $37,44 \pm 6,64$ & $37,79 \pm 4,65 b$ \\
\hline Média & $50,99 \pm 3,71 \mathrm{~A}$ & $53,01 \pm 3,65 \mathrm{~A}$ & \\
\hline CV & \multicolumn{2}{|c|}{30,40} & \\
\hline
\end{tabular}

Médias seguidas de mesma letra, maiúscula na linha e minúscula na coluna, não diferem entre si pelo Teste de Scott Knott, com 5\% de probabilidade. 
A longevidade da progênie fêmea em jejum também foi afetada pela idade da larva hospedeira (Tabela 3). O mesmo náo pode ser dito sobre o número de fêmeas do parasitoide presente na gaiola. Foram mais longevas as fêmeas de D. longicaudata que emergiram das larvas hospedeiras que estavam com oito dias de idade (5,9 dias, em média). As fêmeas provenientes das larvas hospedeiras com seis e sete dias de idade apresentaram a mesma longevidade (em média 4,3 e 3,9 dias, respectivamente), que foram superiores àquelas com cinco dias de idade, as quais viveram cerca de 2,0 dias.

O maior período de sobrevivência das fêmeas obtidas das larvas com a maior idade provavelmente se deve às condiçóes nutricionais desses insetos. Segundo López et al. (2009), o fato de as fêmeas obtidas de larvas de maior idade viverem mais na ausência de alimento pode se dar pelo fato de esses indivíduos possuírem mais reservas de energia do que aqueles provenientes de hospedeiros de menor porte. Esses autores obtiveram resultados semelhantes trabalhando com $D$. longicaudata em larvas de A. fraterculus. Ao avaliarem a expectativa de vida de fêmeas provenientes de larvas pequenas, médias e grandes, observaram que as fêmeas obtidas de larvas de tamanho médio e grande viveram mais que as que foram obtidas de hospedeiro de tamanho pequeno, quando as fêmeas ficaram sem alimento. No entanto, quando o alimento foi fornecido, o tamanho do hospedeiro não influenciou na longevidade das fêmeas. Provavelmente os insetos criados em hospedeiros maiores tiveram mais reservas, mantendo as fêmeas vivas por mais tempo, como no caso do presente trabalho.

Tabela 3. Médias ( \pm Erro Padrão) da longevidade (em jejum) de fêmeas de Diachasmimorpha longicaudata obtidas de larvas de Ceratitis capitata de diferentes idades. Temperatura $26 \pm 1{ }^{\circ} \mathrm{C}$; UR $65 \pm 10 \%$ e fotofase $14 \mathrm{~h}$.

\begin{tabular}{|c|c|c|c|}
\hline \multirow{2}{*}{$\begin{array}{l}\text { Idade } \\
\text { da larva } \\
\text { (dias) }\end{array}$} & \multicolumn{2}{|c|}{$\begin{array}{l}\text { Número de fêmeas por } \\
\text { "unidade de parasitismo" }\end{array}$} & \multirow[t]{2}{*}{ Média } \\
\hline & 1 & 2 & \\
\hline 8 & $5,20 \pm 0,33$ & $6,60 \pm 0,45$ & $5,90 \pm 0,19 a$ \\
\hline 7 & $4,40 \pm 0,08$ & $4,20 \pm 0,33$ & $4,30 \pm 0,17 b$ \\
\hline 6 & $3,80 \pm 0,17$ & $4,00 \pm 0,28$ & $3,90 \pm 0,17 b$ \\
\hline 5 & $2,40 \pm 0,30$ & $2,80 \pm 0,17$ & $2,60 \pm 0,28 c$ \\
\hline Média & $3,95 \pm 0,12 \mathrm{~A}$ & $4,40 \pm 0,16 \mathrm{~A}$ & \\
\hline $\mathrm{CV}$ & \multicolumn{2}{|c|}{11,3} & \\
\hline
\end{tabular}

Médias sequidas de mesma letra, maiúscula na linha e minúsculas na coluna, não diferem entre si pelo teste de Scott Knott, à $5 \%$ de probabilidade.
Em nenhuma das variáveis avaliadas verificou-se a influência da presença de outra fêmea do parasitoide no comportamento dessas fêmeas (Tabelas 1, 2 e 3). Este resultado náo está de acordo com os encontrados por MonToyA et al. (2000), quando observaram que, na presença de membros da mesma espécie, as fêmeas de $D$. longicaudata apresentaram comportamento diferenciado. Neste estudo, os autores avaliaram esses parasitoides individualmente e em grupos de cinco indivíduos, quando ofereceram a eles diferentes proporçóes de larvas de $A$. ludens para um parasitismo de 3 horas. Os autores observaram que, na presença de outros membros da mesma espécie, as fêmeas aumentaram sua atividade de parasitismo. A diferença encontrada entre este trabalho e o de Montoya et al. (2000) pode estar relacionado: à espécie hospedeira utilizada para avaliação; ao número de fêmeas utilizadas, uma vez que grupos de cinco fêmeas podem ter gerado mais superparasitismo, comparado a um grupo de duas fêmeas; e o tempo de exposição, que possivelmente pode ter levado a um incremento no parasitismo.

Os dados obtidos indicam que larvas de C. capitata de sete e oito dias de vida devem ser preferencialmente utilizadas na criação de $D$. longicaudata, pois proporcionaram maior porcentagem de parasitismo e características desejáveis na qualidade dos parasitoides produzidos, como maior número de fêmeas na progênie e mais longevidade das fêmeas da progênie quando da ausência de alimento.

\section{CONCLUSÕES}

A idade das larvas de C. capitata tem efeito sobre a razão sexual, sobre o parasitismo e sobre a longevidade da progênie de D. longicaudata.

\section{AGRADECIMENTOS}

À Fundação de Amparo à Pesquisa de Minas Gerais (FAPEMIG), pelas bolsas de estudo concedidas aos autores (BIPDT e Iniciação científica) e pelo apoio financeiro à pesquisa (APQ-01422-12).

\section{REFERÊNCIAS}

CARVALHO, R.S.; NASCIMENTO, A.S.; MATRANGOLO, W.J.R. Metodologia de criação do parasitóide exótico Diachasmimorpha longicaudata (Hymenoptera: Braconidae), visando estudos em laboratório e em campo. Cruz das Almas: EMBRAPA/CNPMF. 1998. 16p.
CARVALHO, R.S. Estudos de laboratório e de campo com o parasitóide exótico Diachasmimorpha longicaudata Ashmead (Hymenoptera: Braconidae) no Brasil. 2003. 182 f. Tese (Doutorado em Entomologia) Instituto de Biociências, Universidade de São Paulo, São Paulo, 2003. 
DUAN, J.J.; MESSING, R.H. Effects of host substrate and vibration cues on ovipositor-probing behavior in two larval parasitoids of Tephritid Fruit Flies. Journal Insect Behavior, Columbia, v.13, p. 175-186, 2000.

EBEN, A.; BENREY, B.; SIVINSKI, J.; ALUJA, M. Host species and host plant effects on performance of Diachasmimorpha longicaudata (Hymenoptera: Braconidae). Environmental Entomology, Columbia, v.29, p.87-94, 2000 .

FERREIRA, D.F. Sistema de Analise de Variância para dados balanceados. Lavras: Universidade Federal de Lavras. 2000. 66p. (SISVAR 4.1. pacote computacional).

GARCIA, F.R.M. ; RICALDE, M.P. Augmentative biological control using parasitoids for fruit fly management in Brazil. Insects, Basel, v.4, p.55-70, 2012.

GODFRAY, H.C.J. Parasitoids behavioral and evolutionary ecology. New Jersey: Princeton University Press. 1994. 474p.

GODOY, M.J.S; PACHECO, W.S.P.; MALAVASI, A. Moscas-dasfrutas quarentenárias para o Brasil. In: SILVA, R.A.; LEMOS, W.P.; ZUCCHI, R.A. (Eds.). Moscas-das-frutas na Amazônia brasileira: diversidade, hospedeiros e inimigos naturais. Macapá: Embrapa Amapá. 2011. 299p.

LAWRENCE, P.O. Biosteres longicaudatus: development dependence on host (Anastrepha suspensa) physiology. Experimental Parasitollogy, Berlin, v.53, p.396-405, 1982.

LAWRENCE, P.O. The role of 20-hydroxyecdysone in the moulting of Biosteres longicaudatus, a parasite of the Caribbean fruit fly, Anastrepha suspensa. Journal of Insect Physiology, Portici, v.32, p.329-337, 1986.

LÓPEZ, O.P.; HÉNAUT, Y.; CANCINO, J.; LANBIM,M.; CRUZLÓPEZ,L.; ROJAS, J.C. Is host size an indicator of quality in the mass-reared parasitoid Diachasmimorpha longicaudata (Hymenoptera: Braconidae). Florida Entomologist, Gainesville, v.92, p.441-449. 2009.
MONTOYA, P.; LIEDO, P.; BENREY, B.; CANCINO, J.; BARRERA, J.F.; SIVINSKI, J.; ALUJA, M. Functional response and superparasitism by Diachasmimorpha longicaudata (Hymenoptera: Braconidae), a parasitoid of fruit flies (Diptera: Tephritidae). Annals of the Entomological Society of America, Lexington, v.93, p.47-54, 2000.

MONTOYA, P; CACINO, J; PEREZ-LACHAUD, G; LIEDO, P. Host size, superparasitism and sex ratio in mass-reared Diachasmimorpha longicaudata, a fruit fly parasitoid. Biological Control, Lexington, v. 56, p.11-17, 2011

MONTOYA, P; PÉREZ-LACGHAUD, G; LIEDO P. Superparasitism in the fruit fly parasitoid Diachasmimorpha longicaudata (Hymenoptera: Braconidae) and the implications for mass rearing and Augmentative release. Insects, Basel, v.3, p.900-91 1, 2012.

OVRUSKI, S.M.; BEZDJIAN, L.P.; VAN NIEUWENHOVE, G.A.; ALBANOZ-MEDINA, P.; SCHLISERMAN, P. Host preference by Diachasmimorpha longicaudata (Hymneoptera: Braconidae) reared on larvae of Anastrepha fraterculus and Ceratitis capitata (Diptera: Tephritidae). Florida Entomologist, Gainesville, v.94, p.195-200, 2011.

SIVINKI, J.; CALKINS, C.; BARANOWSKI, R.; KHARRIS, D.; BRAMBILA, J.; DIAZ, J; BURNS, R.; HOLLER, T.; DODSON,G. Suppression of a Caribbean fruit fly, Anastrepha suspensa (loew) (Diptera: Tephritidae) population through augmentative release of the parasitoid Diachasmimorpha longicaudata (Ashmead) (Hymenoptera: Braconidae). Biological Control, Lexington, v.56, p.177-185, 1996.

WONG, T.T.Y.; RAMADAN, M.M. Mass rearing biological of larval parasitoids (Hymenoptera: Braconidae: Oppiinae) of tephritid flies (Diptera: Tephritidae) in Hawaii. In "Advances in insect rearing for research and pest management". Florida: Westwiev Press. 1992. $521 \mathrm{p}$.

VAN NIEUWENHOVE, G.A.; OVRUSKI, S.M. Influence of Anastrepha fraterculus (Diptera: Tephritidae) larval instars on the production of Diachasmimorpha longicaudata (Hymenoptera: Braconidae) progeny and their sex ratio. Florida Entomologist, Gainesville, v.94, p.863-868, 2011. 